\title{
Analizando Internet de las Cosas y la nube informática
}

\author{
Fecha de recepción: 2021-11-01 • Fecha de aceptación:2022-01-06 Fecha de publicación: 2022-02-10
}

Darwin Stalin Ramírez Supe ${ }^{1}$

Ministerio de Agricultura y Ganadería stalin_ramirez@yahoo.es https://orcid.org/0000-0003-0568-6489

Estefanía de las Mercedes Zurita Meza² Universidad Técnica de Ambato tefamerce92@gmail.com

https://orcid.org/0000-0001-5509-0152

Francisco Javier Galora Silva ${ }^{3}$ Universidad Internacional de la Rioja, España francisco.galora049@comunidadunir.net https://orcid.org/0000-0002-5464-5336

\section{RESUMEN}

Este artículo presenta un estudio de 2 nuevas tecnologías que han ido creciendo a pasos agigantados en los últimos años, una de ellas es el Internet de las Cosas (IOT), el cual permite a los usuarios conectar miles de millones de máquinas inteligentes e intercambiar información, monitorear y controlar servicios, como sistemas de automatización del hogar interconectados, atención médica, agricultura, monitoreo de seguridad, redes eléctricas o servicios críticos. Mientras que la otra es el Cloud Computing, que se refiere al acceso, configuración y operación de recursos en ubicaciones remotas.

Se propone hacer un análisis de qué es y qué ofrece el loT en los diferentes sensores que existen, de igual forma, analizar los servicios que ofrece el Cloud Computing, y qué resultados se obtendrán si se integran estas 2 nuevas tecnologías, permitiendo que trabajen juntos, logrando 
con ello la generación de nuevas aplicaciones, nuevas áreas de investigación y finalmente lo que a todas las personas les interesa, cómo facilitar la vida humana mediante el uso de estas tecnologías. Concluyendo que la combinación entre el loT y Cloud Computing podrían construir una superpotencia de TI creará tecnologías que actualmente son inimaginables y nuevos campos de investigación que beneficiará a la humanidad.

\title{
PALABRAS CLAVE: Cloud computing, laas, Paas, Saas, Internet de las cosas, sensores
}

\begin{abstract}
This article presents a study of 2 new technologies that have been growing by leaps and bounds in recent years, one of them is the Internet of Things (IoT), which allows users to connect billions of smart machines and exchange information, monitor and control services, such as interconnected home automation systems, health care, agriculture, security monitoring, power grids or critical services. The other is Cloud Computing, which refers to the access, configuration and operation of resources in remote locations.

It is proposed to make an analysis of what loT is and what it offers in the different sensors that exist, likewise, analyze the services offered by Cloud Computing, and what results will be obtained if these 2 new technologies are integrated, allowing them to work together, thereby achieving the generation of new applications, new areas of research and finally what everyone is interested in, how to facilitate human life through the use of these technologies. Concluding that the combination of loT and Cloud Computing could build an IT superpower that will create technologies that are currently unimaginable and new fields of research that will benefit humanity.
\end{abstract}




\section{Introducción}

Con el gran avance tecnológico que se viene dando en los últimos años, se han desarrollado nuevas tecnologías en el área de sistemas informáticos, comunicaciones, robótica, realidad virtual, entre otros, por lo que es muy importante explorar la integración de estas diferentes áreas de trabajo. Como parte de los avances en los sistemas informáticos y las comunicaciones, estos cuentan con tecnologías importantes como la computación en la nube y el Internet de las Cosas (IoT), se puede asegurar que al integrar estas tecnologías se pueden desarrollar nuevos sistemas que pueden facilitar y mejorar la calidad de vida de las personas (Hwang et al., 2013).

El loT permite a los usuarios conectar miles de millones de máquinas inteligentes e intercambiar información, monitorear y controlar servicios, como sistemas de automatización del hogar interconectados, atención médica, agricultura, monitoreo de seguridad, redes eléctricas o servicios críticos. Controlar la infraestructura y controlar el loT son los próximos métodos contemporáneos. Entre ellos, a través de la digitalización dinámica del sistema físico que se prepara, para brindar servicios de valor agregado para dispositivos móviles, la frontera entre el entorno artificial y el entorno real se reduce continuamente. El loT se ejecuta mediante un software especialmente diseñado, que se puede realizar detectando, controlando y cambiando el estado de las cosas (Aazam et al., 2014).

Este descubrimiento que ha generado un gran avance tecnológico para la humanidad, debe convertirse en una serie de objetos interconectados en un mundo conexo, tanto así que permita a los cirujanos realizar operaciones de forma remota, a los usuarios a evaluar sus hogares y proveedores de energía. Debido al uso diversificado de la tecnología loT, el número de dispositivos inteligentes conectados a loT ha proliferado y se espera que supere los 30 millones para finales de 2022. Con el aumento de los elementos de dispositivos heterogéneos asociados con le loT y la generación de datos, será difícil para el loT independiente de aplicar de forma eficaz potencia y ancho de banda a las tareas. Desde este punto de vista, se prevé la integración de la computación en nube y el loT (Alzakholi et al., 2020), cuando la nube está conectada al loT, se genera contenido multimedia.

Dado que el valor multimedia consume más capacidad de procesamiento, espacio, almacenamiento y programación de recursos, será fundamental administrarlo de manera efectiva y realizar una administración eficaz de los recursos en la nube. De lo contrario, los servicios de loT con tareas críticas requieren un procesamiento y una capacidad de respuesta elevados. Recientemente, el loT y la computación en la nube se han estudiado y aplicado constantemente en varios campos, ya que pueden proporcionar un nuevo método para la percepción inteligente y la conexión M2M (incluyendo hombre a hombre, máquina y máquina a máquina), uso bajo demanda e intercambio eficiente de recursos (Dores et al., 2014).

Cuando estas tecnologías funcionan individualmente generan grandes resultados y nuevos campos de investigación, pero si estas dos tecnologías trabajan juntas, los resultados esperados serían asombrosos y se podrían generar soluciones inimaginables que beneficiarían al campo de la ciencia y la investigación. 
En particular, la computación en la nube cobrará cada vez más importancia a través de la ejecución de aplicaciones y un modelo de computación de clase mundial, mientras que la cada vez más ubicua loT será un protagonista tecnológico desde diferentes frentes, incluida la computación en todas partes o la última tecnología, impresoras de última generación, entre otras tecnologías relacionadas con el universo de loT. La Figura 1 muestra una idea de cómo funcionarían la computación en la nube y el loT.

Figura 1.

loT y computación en la nube

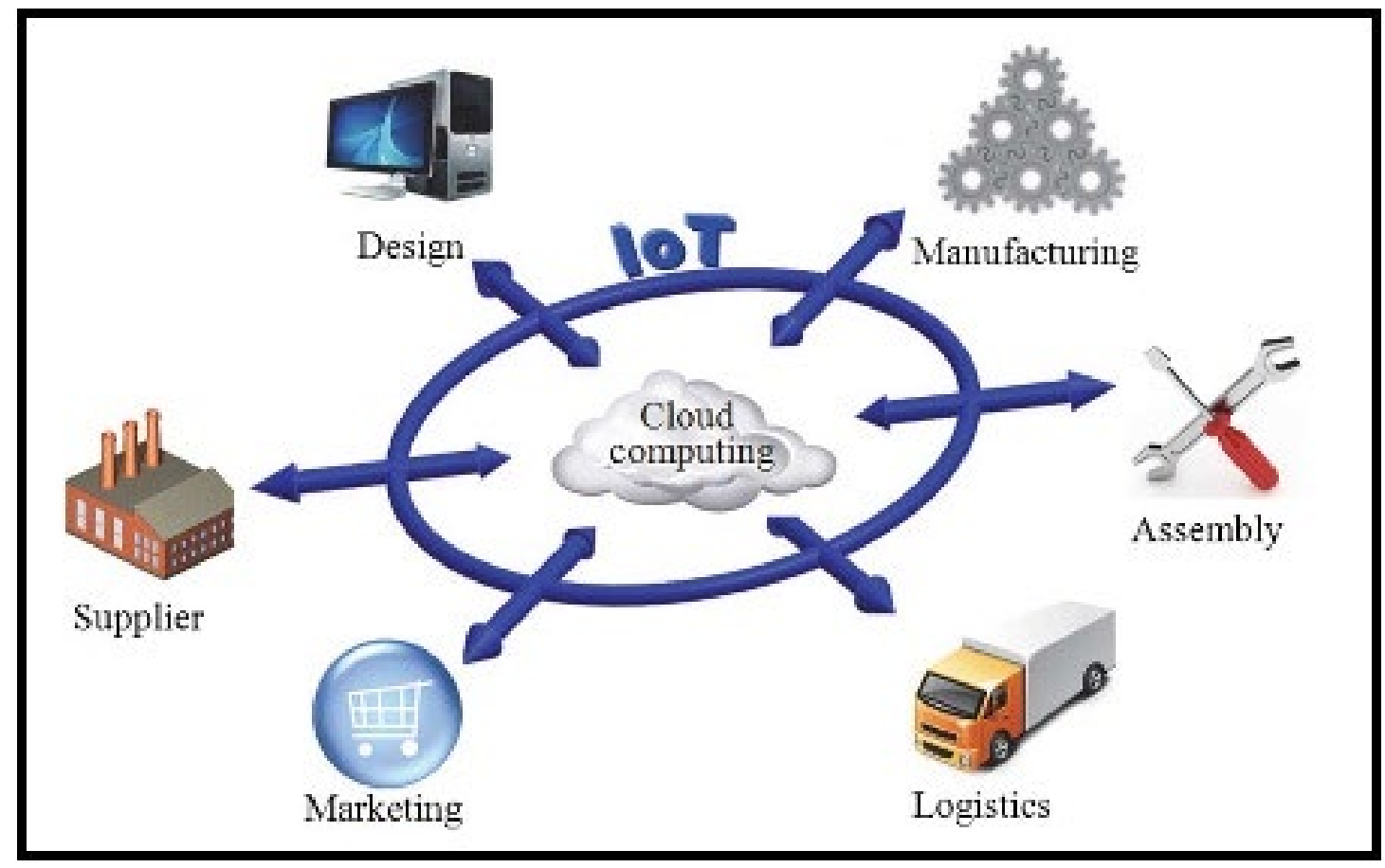

Una de las investigaciones que ha estado trabajando y que incluye estas dos nuevas tecnologías, ha generado el artículo loT y Cloud Computing en Automatización de Sistemas de Modelado de Ensamblaje de los autores Chengen et al. (2014), en el que se propone ayudar a que un sistema de modelado de ensamblajes convencional evolucione hacia un sistema avanzado que sea capaz de hacer frente a la complejidad y los cambios automáticamente.

Además, en el artículo Traer loT y Cloud Computing hacia Pervasive Healthcare de los autores Charalampos Doukas e llias Maglogiannis, se presenta una plataforma basada en Cloud Computing para la gestión de sensores de salud móviles y portátiles, demostrando así el paradigma loT aplicado a el cuidado de la salud, ya que las aplicaciones sanitarias integradas que utilizan redes de sensores corporales generan una gran cantidad de datos que necesitan ser gestionados y almacenados para su procesamiento y uso futuro (Doukas y Maglogiannis, 2012). 
Existen pocos estudios sobre la integración de estas dos importantes nuevas tecnologías, por lo que es necesario realizar un análisis que permita conocer cada una de estas nuevas tecnologías y que se conseguirá haciendo que funcionen juntas.

El presente trabajo está organizado de la siguiente manera. En la sección 2 se habla en detalle de lo que es el loT, en la sección 3 se aborda todo lo relacionado con el Cloud Computing y se hace una integración de estas nuevas tecnologías, luego se presentan las conclusiones y trabajos futuros, y finalmente se detallan las referencias utilizadas en esta investigación.

\section{Metodología}

Los servicios de computación en la nube se implementan en muchos campos relacionados con el IoT, incluido el procesamiento de datos del genoma, la enseñanza y el aprendizaje, los servicios para pymes, los métodos de aprendizaje electrónico, la realidad aumentada, la fabricación, recuperación de emergencias, las ciudades inteligentes, etc., la investigación forense remota, hotelería, negocios, gobierno electrónico y gestión de recursos humanos, Internet del automóvil, entre otros (Perera et al., 2014).

Los desafíos de la computación en nube y el loT en un entorno de aplicación único y separado ha crecido a pasos agigantados y se han generado nuevos escenarios de estudio para los investigadores (Gongjun et al., 2014). La principal dificultad para estudiar cómo integrar loT y la computación en la nube es un descubrimiento incierto. La computación en la nube y loT se han extendido a nivel mundial y su expansión ha sido rápida en los últimos años (Zhang et al., 2010). Cuando se combinan entre sí, las características que muestran serán muy buenas, son muy especiales e importantes entre sí (Aazam et al., 2014). Los investigadores organizaron una serie de aplicaciones relacionadas con la coordinación de la nube y el loT para desarrollar y acumular datos con la ayuda de capacidades informáticas y de almacenamiento en la nube. Las capas de conocimiento de la Figura 2 se explican a continuación. Es importante mencionar que las capas de aplicación, red y detección están interconectadas. 
Figura 2.

Cloud-loT arquitectura

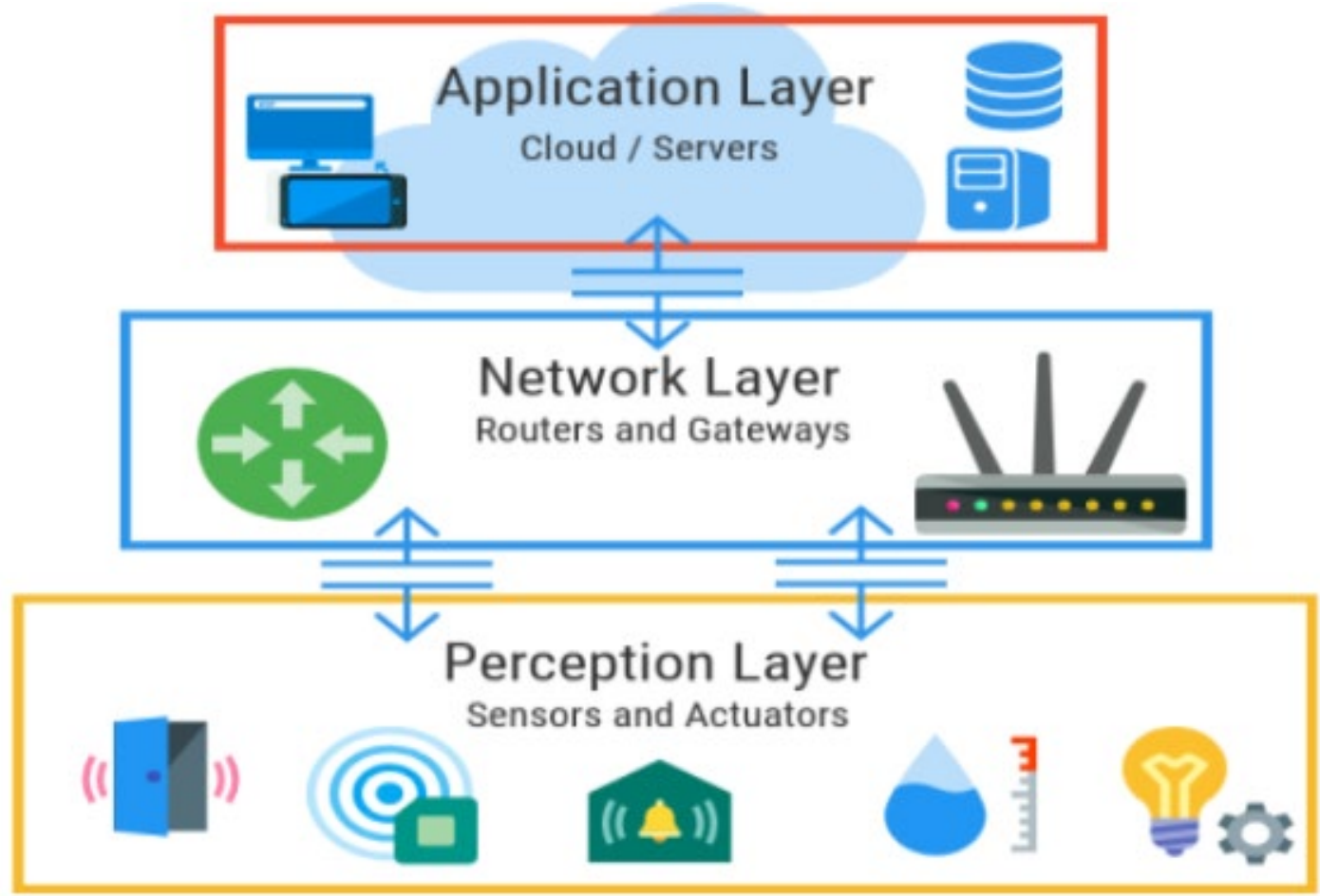

\subsection{Internet de las cosas (IOT)}

El Internet de las cosas (loT) es un nuevo paradigma que se está convirtiendo rápidamente en la piedra angular de las comunicaciones inalámbricas modernas. La idea básica detrás de este concepto es la presencia a nuestro alrededor de diferentes cosas como identificación por radiofrecuencia (RFID), sensores, actuadores, teléfonos celulares, etc., que, al cooperar entre sí, pueden lograr objetivos comunes (Atzori et al., 2010).

Sin duda, el punto fuerte de la loT es el alto impacto que tendrá en diversos aspectos de la vida cotidiana y el comportamiento de las personas. Desde el punto de vista de un usuario privado, los efectos más obvios de la introducción de loT serán visibles, tanto en el lugar de trabajo, como en el hogar. En este contexto, la domótica, la vida asistida, la ciber salud y la mejora del aprendizaje son solo algunos ejemplos de posibles escenarios de aplicación, en los que el nuevo paradigma jugará un papel protagonista en un futuro próximo. Del mismo modo, desde la perspectiva de los negocios usuarios, las consecuencias más aparentes serán igualmente visibles en campos como la automatización y la fabricación industrial, la logística, la gestión de procesos y empresas, el transporte inteligente de personas y mercancías (Gubbi et al., 2013).

Las múltiples definiciones de loT que se pueden rastrear en la comunidad de investigadores dan testimonio del gran interés en el tema de loT y de la vivacidad de los debates al respecto. Al navegar por la literatura, un lector interesado puede experimentar una dificultad real para 
comprender lo que realmente significa loT, qué ideas básicas están detrás de este concepto y qué implicaciones sociales, económicas y técnicas tendrá el despliegue completo de loT (Perera et al., 2014), la Figura 3 muestra claramente su significado.

Figura 3.

Definición de Internet de las cosas

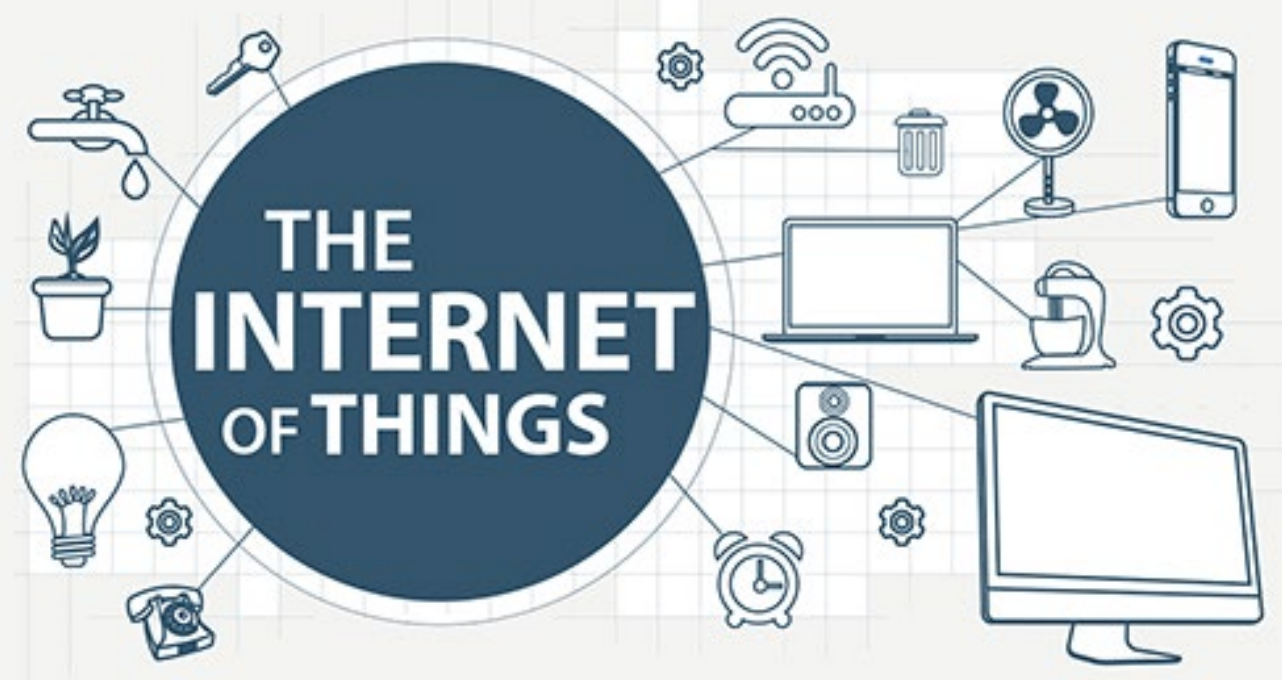

En resumen, loT permite que las personas y los objetos pueden conectarse a Internet, en cualquier lugar y en cualquier momento.

\subsection{Cloud Computing}

La computación en la nube se puede definir como una concepción tecnológica y un modelo de negocio en el que se prestan servicios para el almacenamiento, acceso y uso de recursos informáticos, esencialmente arraigados en la red.

Esta es un paradigma en la forma en que los recursos y las aplicaciones informáticas se utilizan y se entregan como servicios. Estos principales recursos como servicio son la informática, el almacenamiento y la infraestructura de red. La computación en la nube se refiere a proporcionar estos recursos como un servicio a través de Internet al público o en una organización que es de uso privado (Armbrust et al., 2010).

Hay tres tipos de servicios en la nube y difieren en el enfoque sobre cómo se ponen a disposición los recursos. El primer enfoque es hacer que la infraestructura de hardware esté disponible como un servicio, este se denomina Infraestructura como servicio (laaS).

El segundo enfoque es proporcionar una plataforma (el sistema operativo, junto con el software, los marcos y las herramientas necesarios) sobre la infraestructura de hardware, esto se denomina 
Plataforma como servicio (PaaS). El tercer enfoque es proporcionar la aplicación como servicio y se denomina Software como servicio (SaaS) (Emeras et al., 2019). La Figura 4 presenta la idea principal de estos servicios.

Figura 4.

Servicios de computación en la nube

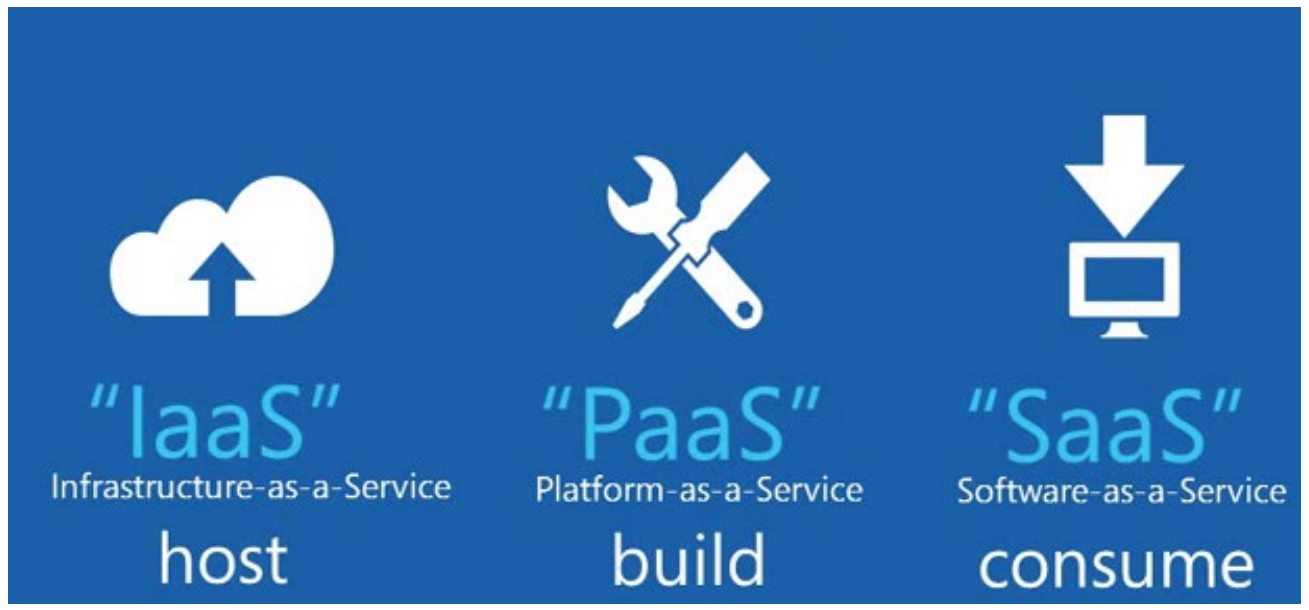

\subsubsection{Infraestructura como servicio laaS}

laaS es la entrega de hardware como servicio. Es una evolución del alojamiento tradicional que no requiere ningún compromiso a largo plazo y permite a los usuarios aprovisionar recursos bajo demanda. El proveedor de laaS hace muy poca administración, además de mantener el centro de datos operativo, los usuarios deben implementar y administrar los servicios de software ellos mismos, tal como lo harían en su propio centro de datos. Amazon Web Services Elastic Compute Cloud (EC2) y Secure Storage Service (S3) son ejemplos de ofertas de laaS (Rajan, 2012).

\subsubsection{Plataforma como servicio PaaS}

PaaS proporciona una plataforma informática que utiliza la infraestructura de la nube. Tiene todas las aplicaciones que normalmente requiere el cliente implementado en él. Por lo tanto, el cliente no tiene que pasar por las molestias de comprar e instalar el software y el hardware necesarios para ello. A través de este servicio, los desarrolladores pueden obtener todos los sistemas y entornos necesarios para el ciclo de vida del software, ya sea en desarrollo, prueba, despliegue y alojamiento de aplicaciones web. Ejemplos clave son GAE, Azure de Microsoft (Jadeja y Modi, 2012).

\subsubsection{Software como servicio SaaS}

SaaS es un modelo en el que un proveedor de servicios aloja las solicitudes y las pone a disposición de los clientes a través de una red, normalmente Internet. SaaS se está convirtiendo en un modelo de entrega cada vez más prevalente a medida que maduran las tecnologías 
subyacentes que respaldan los servicios web y la arquitectura orientada a servicios (SOA) y los nuevos enfoques de desarrollo se vuelven populares. SaaS también se asocia a menudo con un modelo de licencia de suscripción de pago por uso. Las aplicaciones SaaS también deben poder interactuar con otros datos y otras aplicaciones en una variedad igualmente amplia de entornos y plataformas. SaaS está estrechamente relacionado con otros modelos de prestación de servicios que se ha descrito. SaaS se implementa en la mayoría de los casos para proporcionar funcionalidad de software comercial a clientes empresariales a bajo costo, al tiempo que les permite obtener los mismos beneficios del software con licencia comercial y operado internamente sin la complejidad asociada de instalación, administración, soporte y licencias (Godse y Mulik, 2009).

\section{Resultados}

\section{1 loT y cloud computing}

La computación en la nube y el Internet de las cosas (IoT), dos tecnologías completamente diferentes, se han convertido en parte de nuestras vidas. Se espera que su adopción y uso generalizado crezca aún más, convirtiéndolos en componentes importantes de Internet en el futuro.

Esencialmente, la cloud actúa como capa intermedia entre las cosas y las aplicaciones, donde esconde toda la complejidad y las funcionalidades necesarias para implementar estas últimas. Este marco tendrá un impacto en el futuro desarrollo de aplicaciones, donde la recopilación, el procesamiento y la transmisión de información, producirá nuevos desafíos que deben abordarse, también en un entorno de múltiples nubes (Aguzzi et al., 2013), la Tabla 1 muestra la complementariedad e integración de la nube y la loT.

Tabla 1.

Complementariedad e integración de la nube y la IOT

\begin{tabular}{ll}
\hline Internet de las cosas (lot) & Cloud Computing \\
\hline $\begin{array}{l}\text { Ubicuo, cosas colocadas en } \\
\text { todas partes }\end{array}$ & $\begin{array}{l}\text { omnipresente, recursos } \\
\text { utilizables desde todas } \\
\text { partes }\end{array}$ \\
\hline Cosas del mundo real & Recursos virtuales \\
\hline Capacidades & Capacidades \\
computacionales limitadas & $\begin{array}{l}\text { computacionales } \\
\text { virtualmente ilimitadas }\end{array}$ \\
\hline Almacenamiento limitado & capacidades de \\
o sin capacidad de & $\begin{array}{l}\text { almacenamiento } \\
\text { virtualmente ilimitadas }\end{array}$ \\
\hline Intmacenamiento & Internet para la prestación \\
convergencia & de servicios \\
\hline Fuente de un Big Data & Beans para gestionar un Big \\
& Data \\
\hline
\end{tabular}


Las aplicaciones que son posibles o mejoradas significativamente gracias a las tecnologías como loT y Cloud son:

1. Smart healthcare. loT y las tecnologías multimedia han hecho su entrada en el campo de la salud gracias a la vida ambiental asistida y la telemedicina. Los dispositivos inteligentes, Internet móvil y los servicios en la nube, contribuyen a la innovación continua y sistemática de la asistencia sanitaria y permiten servicios médicos ubicuos rentables, eficientes, oportunos y de alta calidad (HU et al., 2017).

2. Smart City. IoT puede brindar un entorno común para servicios de Smart City orientados al futuro (por ejemplo, representaciones 3D a través de sensores RFID y geoetiquetado) y exponiendo la información de manera uniforme. Varias soluciones propuestas recientemente sugieren utilizar arquitecturas de nube para permitir el descubrimiento, la conexión y la integración de sensores y actuadores, creando así plataformas capaces de proporcionar y soportar conectividad ubicua y aplicaciones en tiempo real para ciudades inteligentes (Mitton et al., 2012).

3. Smart home y smart metering. IoT tiene una gran aplicación en entornos domésticos, donde los dispositivos integrados heterogéneos permiten la automatización de actividades internas comunes. En este escenario, la nube es el mejor candidato para crear aplicaciones flexibles con solo unas pocas líneas de código, lo que hace que la automatización del hogar sea una tarea trivial (Kamilaris et al., 2011). Para permitir que una variedad de hogares inteligentes unifamiliares independientes accedan a servicios reutilizables a través de Internet, la solución resultante debe satisfacer tres requisitos cruciales: interconexión de red interna (es decir, cada dispositivo digital en el hogar inteligente debe poder interconectarse con cualquier otro), control remoto inteligente (es decir, los electrodomésticos y servicios en el hogar inteligente deben ser gestionados de manera inteligente por cualquier dispositivo, desde cualquier lugar) y automatización (los electrodomésticos interconectados dentro del hogar deben implementar sus funciones mediante la vinculación a los servicios proporcionados por la nube orientada al hogar inteligente) (Wu He et al., 2014).

4. Video vigilancia. La videovigilancia inteligente se ha convertido en una herramienta de la mayor importancia para varias aplicaciones relacionadas con la seguridad. Como alternativa a los sistemas de gestión autónomos internos, los análisis de video complejos requieren soluciones basadas en la nube para satisfacer adecuadamente los requisitos de almacenamiento (por ejemplo, los medios almacenados son centralmente seguros, tolerantes a fallas, bajo demanda, escalables y accesibles a alta velocidad) y procesamiento (por ejemplo, procesamiento de video, algoritmos de visión por computadora y módulos de reconocimiento de patrones para extraer conocimiento de las escenas). Las soluciones propuestas almacenan y administran de manera inteligente el contenido de video proveniente de cámaras (IP y analógicas) y lo entregan de manera eficiente a múltiples dispositivos de usuario a través de Internet, mediante la distribución de las tareas de procesamiento a través de los recursos del servidor físico bajo demanda, de una manera equilibrada de carga y tolerante a fallas (Held et al., 2012).

5. Movilidad inteligente y automotriz. Como tecnología emergente, se espera que loT ofrezca soluciones prometedoras para transformar los sistemas de transporte y los 
servicios de automóviles (es decir, sistemas de transporte inteligentes, ITS). La integración de tecnologías en la nube con WSN, RFID, redes satelitales y otras tecnologías de transporte inteligente representa una oportunidad prometedora para abordar los principales desafíos actuales. Se puede desarrollar e implementar una nueva generación de nubes de datos vehiculares basadas en loT para brindar muchos beneficios comerciales, como aumentar la seguridad vial, reducir la congestión vial, administrar el tráfico y recomendar el mantenimiento o reparación de los automóviles (Wu He et al., 2014).

\subsection{Desafíos Cloud-loT}

La capa intermedia entre los objetos y las aplicaciones es el almacenamiento en la nube, que oculta matices y funciones. Todos reconocemos que Internet de las Cosas es una red de artefactos enlazados, y en estos objetos están implicadas varias aplicaciones. Los problemas de cada aplicación son únicos, pero generalmente pertenecen a categorías similares. Para resolver estos desafíos se debe prestar más atención a los desafíos de seguridad y evaluar las consecuencias de las nuevas tecnologías. Después de integrar la nube y el Internet de las Cosas, la gente ha estado preocupada por la desconfianza y comprensión de los proveedores de la nube, sobre la ubicación física de la información detallada transmitida a la nube, a través de diferentes protocolos de loT. Hay varias preguntas sobre el sistema de almacenamiento de servicios en la nube de múltiples inquilinos. La información de los consumidores múltiples se almacena en una instalación, lo que destruirá la confidencialidad y el proceso de fuga de información confidencial. Debido a la desconfianza en los proveedores de servicios en la nube, esta forma de vulnerabilidad se considera una amenaza interna y es, con mucho, uno de los problemas más imprevistos en la industria de TI. Los desafíos clave de Cloud-IoT se explican a continuación.

A. Seguridad. Los datos seguros de loT se colocan en la nube para su procesamiento y recuperación. Esto implica el cifrado de datos y la seguridad de los datos que se envían o almacenan en repositorios basados en la nube, durante el acceso y uso de la nube. El grado de falta de información de computación en la nube hace que los propietarios de los datos no sean conscientes de la ubicación física de sus datos. Hoy en día, los datos están estrechamente relacionados con todo lo que nos rodea, por lo que la seguridad de los datos en el paradigma Cloud-IoT es el tema principal.

B. Rendimiento computacional y almacenamiento. Los planes de rendimiento de almacenamiento y computación que incluyen el uso de dispositivos loT basados en la nube requieren requisitos de objetivos de alto rendimiento. Dado que los dispositivos loT basados en la nube funcionan en muchas aplicaciones, es difícil cumplir con estas especificaciones en todos los entornos.

C. Fiabilidad. Los dispositivos de loT dependen de la nube para proporcionar trabajo para aplicaciones de tiempo crítico, y el efecto reflejará directamente el resultado del programa. Por ejemplo, en el campo de los instrumentos quirúrgicos para automóviles o la seguridad. 
D. Almacenamiento de Big Data. Alrededor de 2025 se proporcionarán casi 50 mil millones de dispositivos loT, y estos ingresos se convertirán en un obstáculo importante para que los proveedores de servicios en la nube accedan a los datos de forma rápida y segura.

E. Mantenimiento. Con base en el conocimiento aprendido en las secciones anteriores, se necesitan tecnologías y planes extremadamente eficientes para rastrear y administrar la protección y la eficiencia en el entorno de la nube, para satisfacer la demanda de hasta 50 mil millones de dispositivos loT.

F. Computación al límite. Las restricciones de latencia, las restricciones de movilidad y la implementación de loT distribuida de Geodis, requieren que la nube responda de inmediato. Por lo tanto, la computación en el borde es un compromiso entre la computación clásica y la computación en la nube. Aunque está más cerca de la implementación, es difícil fusionarse porque requiere conocimiento de la ubicación.

G. Dispositivos de loT asistidos por el usuario. En una implementación de loT de este tipo, los usuarios deben incluir detalles y beneficios para compensar su participación en la comunicación. En el arduo desafío desde que empezaron a funcionar los factores sociales, el consumidor ha hecho aportes en su contexto.

H. Interacción con los dispositivos. Los sistemas de Cloud loT generalmente requieren información de varios dispositivos para su procesamiento e implementación. En este caso, las especificaciones, como el espacio de almacenamiento y la potencia informática basada en la nube, pueden resultar difíciles.

\section{Conclusiones}

Como parte de las conclusiones del presente artículo, se determina que la combinación de Internet de las cosas (IoT) y la computación en la nube, así como los servicios de desarrollo, podrían construir una superpotencia de TI que creará tecnologías que actualmente son inimaginables, y nuevos campos de investigación que beneficiará a toda la humanidad, sin importar si están involucrados en el mundo de la tecnología o trabajan en otra zona.

Tanto el loT como el Cloud Computing son tecnologías que irán creciendo y evolucionando en el futuro, ya que hoy en día uno de los mayores obstáculos es el canal de comunicación entre ellos, por que a mayor demanda de peticiones, se necesita mayor ancho de banda, por ende, con la llegada de las conexiones $5 \mathrm{G}$, permitirá el crecimiento y desarrollo de las tecnologías habladas en el documento presente.

Con este análisis se podrá tener una idea clara de cómo funciona el Internet de las cosas y el Cloud Computing, de esta forma, en el futuro se puede realizar un estudio que proponga utilizar estas 2 tecnologías y que beneficie a la sociedad, esto no será una tarea fácil, pero con un poco de esfuerzo e inversión se puede lograr. 


\section{Referencias}

Aazam, M., Khan, I., Alsaffar, A. A. y Huh, E.-N. (2014). Cloud of Things: Integrating Internet of Things and cloud computing and the issues involved. En 2014 11th International Bhurban Conference on Applied Sciences and Technology (IBCAST). IEEE. https://doi.org/10.1109/ibcast.2014.6778179

Aguzzi, S., Bradshaw, D., Canning, M., Cansfield, M., Carter, P., Cattaneo, G., \& Stevens, R. (2013). Definition of a research and innovation policy leveraging cloud computing and loT combination. Final Report, European Commission, SMART, 37, 2013.

Alzakholi, O., Haji, L., Shukur, H., Zebari, R., Abas, S. y Sadeeq, M. (2020). Comparison Among Cloud Technologies and Cloud Performance. Journal of Applied Science and Technology Trends, 1(2), 40-47. https:// doi.org/10.38094/jastt1219

Armbrust, M., Fox, A., Griffith, R., Joseph, A. D., Katz, R., Konwinski, A., Lee, G., Patterson, D., Rabkin, A., Stoica, I. y Zaharia, M. (2010). A view of cloud computing. Communications of the ACM, 53(4), 50-58. https:// doi.org/10.1145/1721654.1721672

Atzori, L., lera, A., \& Morabito, G. (2010). The internet of things: A survey. Computer networks, 54(15), 27872805. https://doi.org/10.1016/j.comnet.2010.05.010

Chengen Wang, Zhuming Bi y Li Da Xu. (2014). loT and Cloud Computing in Automation of Assembly Modeling Systems. IEEE Transactions on Industrial Informatics, 10(2), 1426-1434. https://doi.org/10.1109/ tii.2014.2300346

Dores, C., Reis, L. P., \& Lopes, N. V. (2014). Internet of things and cloud computing. In 2014 9th Iberian Conference on Information Systems and Technologies (CISTI) (pp. 1-4). IEEE. DOI: 10.1109/CISTI.2014.6877071

Doukas, C., \& Maglogiannis, I. (2012). Bringing loT and cloud computing towards pervasive healthcare. In 2012 Sixth International Conference on Innovative Mobile and Internet Services in Ubiquitous Computing (pp. 922-926). IEEE. DOI: 10.1109/IMIS.2012.26

Emeras, J., Varrette, S., Plugaru, V. y Bouvry, P. (2019). Amazon Elastic Compute Cloud (EC2) versus In-House HPC Platform: A Cost Analysis. IEEE Transactions on Cloud Computing, 7(2), 456-468. https://doi. org/10.1109/tcc.2016.2628371

Godse, M., \& Mulik, S. (2009,). An approach for selecting software-as-a-service (SaaS) product. In 2009 IEEE International Conference on Cloud Computing (pp. 155-158). IEEE.

Gubbi, J., Buyya, R., Marusic, S., \& Palaniswami, M. (2013). Internet of Things (IoT): A vision, architectural elements, and future directions. Future generation computer systems, 29(7), 1645-1660. https://doi.org/10.1016/j.future.2013.01.010 
Held, C., Krumm, J., Markel, P. y Schenke, R. P. (2012). Intelligent Video Surveillance. Computer, 45(3), 8384. https://doi.org/10.1109/mc.2012.97

Hu, Q.-H., Zhang, S., \& Han, Y. (2017). Research of Smart Home Service Platform Based on Mobile Cloud. DEStech Transactions on Engineering and Technology Research, (sste). https://doi.org/10.12783/dtetr/ sste2016/6503

Hwang, K., Dongarra, J. y Fox, G. C. (2013). Distributed and cloud computing: from parallel processing to the internet of things. Morgan Kaufmann.

Jadeja, Y., \& Modi, K. (2012). Cloud computing-concepts, architecture and challenges. In 2012 International Conference on Computing, Electronics and Electrical Technologies (ICCEET) (pp. 877-880). IEEE.

Kamilaris, A., Pitsillides, A., \& Trifa, V. (2011). The smart home meets the web of things. International Journal of Ad Hoc and Ubiquitous Computing, 7(3), 145-154. https://doi.org/10.1504/ijahuc.2011.040115

Mitton, N., Papavassiliou, S., Puliafito, A., \& Trivedi, K. S. (2012). Combining Cloud and sensors in a smart city environment. EURASIP journal on Wireless Communications and Networking, 2012(1), 1-10. https://doi. org/10.1186/1687-1499-2012-247

Perera, C., Zaslavsky, A., Christen, P., \& Georgakopoulos, D. (2013). Context aware computing for the internet of things: A survey. IEEE communications surveys \& tutorials, 16(1), 414-454. https://doi.org/10.1109/ surv.2013.042313.00197

Rajan, R. A. P. (2012). Evolution of Cloud Storage as Cloud Computing Infrastructure Service. IOSR Journal of Computer Engineering, 1(1), 38-45. https://doi.org/10.9790/0661-0113845

Wu He, Gongjun Yan., \& Li Da Xu. (2014). Developing Vehicular Data Cloud Services in the loT Environment. In IEEE Transactions on Industrial Informatics, 10(2), 1587-1595. https://doi.org/10.1109/tii.2014.2299233

Zhang, Q., Cheng, L., \& Boutaba, R. (2010). Cloud computing: state-of-the-art and research challenges. Journal of Internet Services and Applications, 1(1), 7-18. https://doi.org/10.1007/s13174-010-0007-6 
Copyright (c) 2022 Darwin Stalin Ramírez Supe, Estefanía de las Mercedes Zurita Meza y Francisco Javier Galora Silva

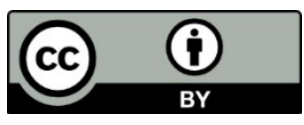

Este texto está protegido bajo una licencia internacional Creative Commons 4.0.

Usted es libre para Compartir-copiar y redistribuir el material en cualquier medio o formato - y Adaptar el documento - remezclar, transformar y crear a partir del material-para cualquier propósito, incluso para fines comerciales, siempre que cumpla las condiciones de Atribución. Usted debe dar crédito a la obra original de manera adecuada, proporcionar un enlace a la licencia, e indicar si se han realizado cambios. Puede hacerlo en cualquier forma razonable, pero no de forma tal que sugiera que tiene el apoyo del licenciante o lo recibe por el uso que hace de la obra.

$\underline{\text { Resumen de licencia - Texto completo de la licencia }}$ 